\title{
Droit communautaire et transport des matières dangereuses*
}

\author{
P. BOURGEOIS*
}

(Manuscrit reçu le 4 juin 1992)

RÉSUMÉ La Commission des Communautés européennes a pris acte de l'existence d'une réglementation internationale pour le transport de matières dangereuses, dite "ADR". Dans un premier temps, elle n'envisage pas d'élaborer une réglementation communautaire mais préconise l'adoption, dans un but d'uniformisation, par tous les Etats membres de l'ADR comme réglementation nationale. C'est chose faite en France depuis la parution au Journal officiel du 13 octobre 1992 de l'arrêté du 15 septembre 1992. La France a conservé, toutefois, certaines spécificités dont l'abandon aurait conduit à une baisse sensible du niveau de sécurité. La Communauté a complété, néanmoins, la réglementation ADR par des dispositions concernant la formation professionnelle, l'accès à la profession de transporteur routier, le cabotage routier.

ABSTRACT The Commission of the European communities has taken formal note of the socalled "ADR" international recommendations on the transport of dangerous goods. In a first stage, the Commission will not draw up a Community law, but, with a view to standarzation, it recommends the adoption of $A D R$ as a national regulation by all member States. This has been enforced in France since the publication of an order of September 15, 1992 in the "Journal officiel" of October 13, 1992. France, however, has maintained some specific provisions whose renunciation would have led to a sensible decline of the safety level. The Commission has nevertheless amplified the ADR by provisions about vocational training, access to the profession of road transport operator, road cabotage.

\section{INTRODUCTION}

Le transport des matières dangereuses en Europe est soumis à des impératifs contradictoires :

- la nécessité d'assurer la libre circulation des marchandises et des personnes ;

- la nécessité de renforcer la sécurité et la protection de l'environnement pour répondre aux attentes de l'opinion publique ;

* Communication présentée lors des Journées SFRP, Paris, 2 et 3 avril 1992, "Le nouveau règlement pour le transport des matières dangereuses et radioactives".

** Ministère de l'équipement, du logement, des transports, Direction des transports terrestres, Mission du transport des matières dangereuses, 92055 Paris La Défense Cedex 04. 
- la nécessité de tenir compte de la diversité des conditions économiques et juridiques.

L'existence d'accords internationaux permet heureusement de régler une grande partie des problèmes, mais la perspective du marché unifié de 1993 a conduit la Commission des Communautés européennes à mieux préciser sa position au cours des dernières années. Constatant la pertinence des instruments internationaux, la Commission a d'abord recommandé leur utilisation sur des bases harmonisées pour les règlements nationaux tout en prévoyant de fixer, si nécessaire, au niveau communautaire des mesures complémentaires propres à améliorer la sécurité du transport des matières dangereuses quel que soit le mode concerné.

\section{Le contexte communautaire actuel}

Partant de cette position, la Commission a adopté les mesures suivantes :

1) La directive $89 / 684$ concernant la formation professionnelle des conducteurs de véhicules [2]. Cette directive devrait être prochainement complétée par une harmonisation des systèmes de formation.

2) La directive 89/438 concernant l'accès à la profession de transporteur routier [3]. Cette directive fixe des exigences quant à la connaissance de la réglementation du transport des matières dangereuses.

3) Le règlement 4059/89 concernant le cabotage routier [4]. Ce règlement permet aux Etats membres d'imposer une partie de leur réglementation nationale relative au transport des matières dangereuses, mais il précise que les normes techniques auxquelles doivent satisfaire les véhicules utilisés pour effectuer les opérations de cabotage sont celles qui sont imposées aux véhicules admis à la circulation en transport international.

Des projets sont en cours d'élaboration par la Direction générale VII, ils concernent :

1) la désignation d'un préposé à la sécurité,

2) la signalisation et l'information nécessaire à la prévention des risques,

3) les organismes génétiquement modifiés.

D'autres Directions générales prévoient également des textes qui auront une incidence sur la réglementation du transport des matières dangereuses :

1) la directive sur la récupération des vapeurs d'hydrocarbures,

2) l'extension du champ de la directive SEVESO aux gares et ports,

3) la directive sur les appareils à pression qui s'appliquera aux matériels de transport. 
Il est également bon de rappeler la doctrine de la Commission concernant la reconnaissance mutuelle entre Etats membres des normes techniques. Le principe de reconnaissance interdit à un Etat membre importateur de soumettre à de nouveaux contrôles des produits qui ont déjà satisfait aux mêmes contrôles dans un autre Etat membre. II est assorti d'une certaine atténuation par l'affirmation d'une nécessité de compétence pour les organismes et laboratoires qui ont procédé aux contrôles.

Ce rapide panorama montre que l'action de la Commission est loin d'être négligeable au-delà de son affirmation de la pertinence des accords internationaux concernant le transport des matières dangereuses. Ces accords, qui comportent inévitablement des lacunes ou des possibilités d'interprétation, doivent donc être intégrés au mieux dans le contexte communautaire sous l'égide de la Commission, mais avec une participation des experts des Etats membres.

\section{La position de la France}

La sécurité du transport des matières dangereuses repose sur deux piliers :

- une réglementation qui édicte des prescriptions techniques ou administratives de nature à minimiser les risques pour les personnes et pour l'environnement ;

- des comportements et des pratiques professionnelles qui garantissent la fiabilité et le respect des procédures.

Par ailleurs, les pouvoirs publics peuvent définir des politiques de transport concernant les règles de circulation ou de stationnement, l'exploitation optimale de chaque mode, les conditions de chargement et de déchargement dans les établissements industriels, etc.

Une politique communautaire devrait prendre en compte l'ensemble des éléments qui viennent d'être schématisés (une énumération complète serait longue et même hasardeuse). Elle devrait, en plus, distinguer le domaine du transport proprement dit qui soulève moins de difficultés et le domaine de la mise sur le marché qui en soulève beaucoup compte tenu des imprécisions des accords internationaux.

Les actions menées par la France soit au plan national, soit au plan international, essaient de tenir compte de telles perspectives.

\subsection{Au plan national}

La France vient de procéder à la réécriture de la réglementation du transport routier des matières dangereuses. Elle a adopté pour ce faire I'ADR comme texte de base [1] en se bornant à un minimum de prescriptions différentes qui pourraient être considérées comme des entraves 
techniques à la mise sur le marché au sens de la directive 83/169 (le projet de règlement a d'ailleurs été notifié à la Commission, le délai de statu quo expirant le 17 février). Ces prescriptions complémentaires sont, la plupart du temps, des précisions apportées au texte de I'ADR lorsque celui-ci le nécessite, mais il était difficile de tirer un trait complet sur 40 ans de réglementation et quelques particularités subsistent :

- les matières transportées à une température supérieure à $80^{\circ} \mathrm{C}$ relèvent de la réglementation (prescription adoptée à la suite d'accidents dans l'épandage des bitumes) ;

- en attendant la directive "appareil à pression", la réglementation française des appareils à pression s'applique pour les récipients de capacité inférieure à 4501 ;

- les transports alternés ou simultanés de matières dangereuses et de denrées alimentaires sont interdits ;

- l'équipement électrique prescrit à l'appendice B.2 s'impose à toutes les unités de transport ;

- l'emploi de l'acier T1 est interdit (prescription un peu désuète, liée à l'accident de Los Alfaquès) ;

- les règles d'étiquetage sont un peu plus strictes;

- des prescriptions administratives sont édictées pour le chargement et le déchargement.

Ces particularités ne sont pas de nature à mettre en cause la "marche en avant" vers l'ADR accomplie par la France. Certaines sont d'ailleurs appelées à disparaître avec l'évolution de la réglementation internationale.

Les points importants à mettre en évidence sont de deux ordres :

- les prescriptions relevant de l'autorité compétente ne peuvent être éludées et chaque Etat membre édicte les siennes ; on reviendra sur ce point ;

- le transport des matières les plus dangereuses ne peut être effectué que par des entreprises titulaires d'un certificat d'assurance de la qualité sur la base de la norme ISO 9002 [6]. II s'agit là d'une mesure demandée depuis longtemps par l'opinion publique et qui s'apparente aux restrictions introduites par l'article 7 du GGVS (autorisation de transport ou utilisation de citernes renforcées pour le transport des liquides inflammables).

Il est également à noter que la France impose des procédures d'assurance de la qualité pour les emballages, ce qui rejoint une prescription identique en Allemagne.

L'application de la nomme ISO 9002 au transport combiné et par là même au transport ferroviaire est à l'étude. 


\subsection{Au plan international}

La France manifeste depuis plusieurs années son souhait de mise en œuvre d'une politique communautaire pour le transport des matières dangereuses. Cette politique devrait concerner l'application harmonisée des accords internationaux, les études et les mesures de sécurité complémentaires.

\section{1) L'application harmonisée des accords internationaux}

Concernant le transport proprement dit, la France a, désormais, la même interprétation que l'Allemagne de l'article 5 du règlement 4059/89 (elle maintient simplement l'interdiction de l'acier $\mathrm{T} 1$ à titre symbolique et l'obligation du limiteur de vitesse qui va devenir européenne) [3].

Concernant l'application des prescriptions de l'autorité compétente au titre de l'annexe B de l'ADR, elle se réfère à la "nouvelle approche" de la Commission pour faire appel à la normalisation comme preuve de conformité harmonisée aux exigences essentielles de l'ADR (qui, à terme, devrait être réécrit dans la perspective "nouvelle approche"). La récente création du TC 296 au Comité européen de normalisation justifie cette démarche.

La DG III mène une démarche analogue au travers de la directive "appareil à pression supérieure à 0,5 bar". Comme les autres Etats membres, la France veillera à la cohérence de cette directive et de I'ADR. La normalisation constituera un mode de preuve commun aux deux textes.

\section{2) Dispositions complémentaires}

La certification d'assurance de la qualité évoquée plus haut se réfère à une pratique usuelle entre professionnels. La nomme ISO 9002 ne fixe pas, par nature, un niveau de performance ou de sécurité. Cette lacune a été reconnue au niveau européen. Un groupe d'études a été créé au Comité européen de normalisation pour proposer des solutions.

Sur le plan de la Commission (ou sur le plan de l'ADR pour la partie technique), des réflexions sont à mener notamment pour améliorer la stabilité des véhicules, pour améliorer le mode de calcul des citernes en se référant aux situations accidentelles, pour une politique de transport multimodale, pour harmoniser les conditions de contrôle.

\section{RÉFÉRENCES}

[1] Accord européen relatif au transport international des marchandises dangereuses par route $(A D R)$ et protocole de signature en date à Genève, du 30 septembre 1957 (Documents administratifs, 7). Paris : Journal officiel, 1991. 
[2] COMMUNAUTES EUROPEENNES - Directive du Conseil, du 21 décembre 1989, concernant la formation personnelle de certains conducteurs transportant des marchandises par route (89/684/CEE). J.O.C.E., 1989, L 398, p. 33.

[3] COMMUNAUTES EUROPEENNES - Directive du Conseil, du 21 juin 1989, modifiant la directive 74/561/CEE concernant l'accès à la profession de transporteur de marchandises par route dans le domaine des transports nationaux et internationaux, la directive 74/562/CEE concernant l'accès à la profession de transporteur de voyageurs par route dans le domaine des transports nationaux et internationaux et la directive 77/796/CEE visant à la reconnaissance mutuelle des diplômes, certificats et autres titres de transporteur de marchandises et de transporteur de personnes par route et comportant des mesures destinées à favoriser l'exercice effectif de la liberté d'établissement des transporteurs (84/438/CEE). J.O.C.E, 1989, L 212, p. 101.

[4] COMMUNAUTES EUROPEENNES - Règlement $n^{\circ} 4059 / 89$ du Conseil, du 21 décembre 1989, fixant les conditions de l'admission de transporteurs non résidents aux transports nationaux de marchandise par route dans un Etat membre. J.O.C.E., 1989, L 390 , p. 3.

[5] FRANCE - Arrêté du 15 septembre 1992 relatif au règlement pour le transport des matières dangereuses (prescriptions routières et Nomenclature alphabétique des matières) (matières dangereuses 1992 n $^{\circ}$ 1). J.O.R.F., 13 octobre 1992, p. 14257.

[6] INTERNATIONAL STANDARD ORGANISATION (ISO) - Système qualité. Modèle pour l'assurance de la qualité en production et installation (ISO 9002). Genève : ISO, 1987. (AFNOR EN 29002). Paris-La Défense: AFNOR, 1988. 